\title{
Ultrasonography-guided ilioinguinal-iliohypogastric nerve block for inguinal herniotomies in ex-premature neonates
}

Shuying Lee $\underline{L}^{1}$, MMed, FANZCA, Josephine Swee Kim $\underline{\operatorname{Tan}}^{1}$, MMed

\begin{abstract}
The ilioinguinal-iliohypogastric (IG-IH) nerve block provides effective opioid-sparing analgesia for inguinal surgeries. The technique is especially useful in apnoea-prone premature neonates with sacral anomalies and coagulopathy. A recent retrospective review of 82 ex-premature neonates who underwent inguinal herniotomy at KK Women's and Children's Hospital, Singapore, reported a success rate of $89 \%$ for landmark-guided IG-IH blocks. All blocks in that study were performed by senior paediatric anaesthetists using the landmark-based technique, which relies on fascial clicks. The IG-IH block is expected to be technically more difficult in neonates. There is also a stronger need to ensure success in these patients in order to avoid the use of opioids and reduce the risk of postoperative apnoea. Ultrasonographic guidance has been reported to improve the success of IG-IH blocks in older children to up to $94 \%$. Herein, we report a series of six ex-premature neonates in whom ultrasonography-guided IG-IH blocks were successfully performed using reduced volumes of local anaesthetics (mean volume $0.17 \mathrm{~mL} / \mathrm{kg}$ ) for inguinal herniotomy.
\end{abstract}

Keywords: ilioinguinal block, inguinal herniotomy, premature neonate, ultrasound

\section{INTRODUCTION}

Inguinal herniotomies on ex-premature neonates are a challenge for both the anaesthetists and surgeons involved. Postoperative apnoea occurs in $20 \%-50 \%$ of preterm infants. ${ }^{(1)}$ The ideal anaesthetic technique aims to provide optimal operating conditions and superior analgesia with minimal opioid usage, while avoiding postoperative apnoea and block-related complications. Current practice in our institution involves the use of regional anaesthesia (i.e. sole spinal, caudal or ilioinguinal and iliohypogastric nerve block) with or without general anaesthesia. In the presence of cutaneous stigmata for spina bifida, ultrasonography may be used to locate the tip of the dural sac before proceeding with a caudal block to mitigate the risk of dural puncture. ${ }^{(2)}$ A safer alternative would be to perform an ilioinguinal-iliohypogastric (IG-IH) nerve block. As it is a peripheral nerve block, it is associated with less risks of serious sequelae, ${ }^{(3)}$ such as inadvertent intravascular injection or dural puncture. This is especially relevant in neonates, in whom the dural sac ends at a lower position. It is also suitable in neonates with significant coagulopathy.

In a recent retrospective review of 82 ex-premature neonates who underwent inguinal herniotomy in our unit at the Department of Paediatric Anaesthesia, KK Women's and Children's Hospital, Singapore, landmark-based IG-IH nerve blocks were reported to have good success rates (up to $89 \%$ ). ${ }^{(4)}$ However, all the IG-IH blocks were performed by experienced anaesthetists who regularly performed this procedure. As anatomical surface landmarks vary with growth and tend to be poorly defined in younger children, accurate placement of local anaesthetics (LA) using landmark-based techniques is seldom possible in these patients. ${ }^{(5)}$ In fact, a study by Weintraud et al found that LA was often inaccurately placed in adjacent structures (e.g. the peritoneum) in children, resulting in unpredictable block results. ${ }^{(6)}$ Furthermore, as it is more difficult to appreciate fascial clicks in the thin-walled abdomen of neonates, the likelihood of complications such as bowel puncture and pelvic haematoma is higher. The use of larger volumes of LA, in an attempt to increase the success rates in these patients, may be contraindicated due to possible complications such as LA toxicity and inadvertent femoral nerve block (up to $9 \%$ ). ${ }^{(7)}$

In older children, ultrasonographic guidance has been shown to improve success rates ${ }^{(8)}$ and reduce LA requirements ${ }^{(9)}$ in IG-IH nerve blocks. In this study, we report our experience in performing ultrasonography-guided IG-IH blocks in six preterm neonates who presented for inguinal herniotomies.

\section{CASE REPORT}

Six premature neonates ( 4 female, 2 male), with a mean postconceptual age of 38.6 weeks and mean weight of $2.3 \mathrm{~kg}$, were recruited for the study following approval from the hospital ethics committee. Written informed consent was obtained from the parents.

The mean preoperative haemoglobin level was $10.6 \mathrm{~g} / \mathrm{dL}$. Four neonates had no pre-existing respiratory dysfunction, but two neonates had hyaline membrane disease and were on

${ }^{1}$ Department of Paediatric Anaesthesia, KK Women's and Children's Hospital, Singapore

Correspondence: Dr Shuying Lee, Consultant, Department of Paediatric Anaesthesia, KK Women's and Children's Hospital, 100 Bukit Timah Road, Children'S Tower Level 2, Singapore 229899. leeshuying79@yahoo.com.sg 

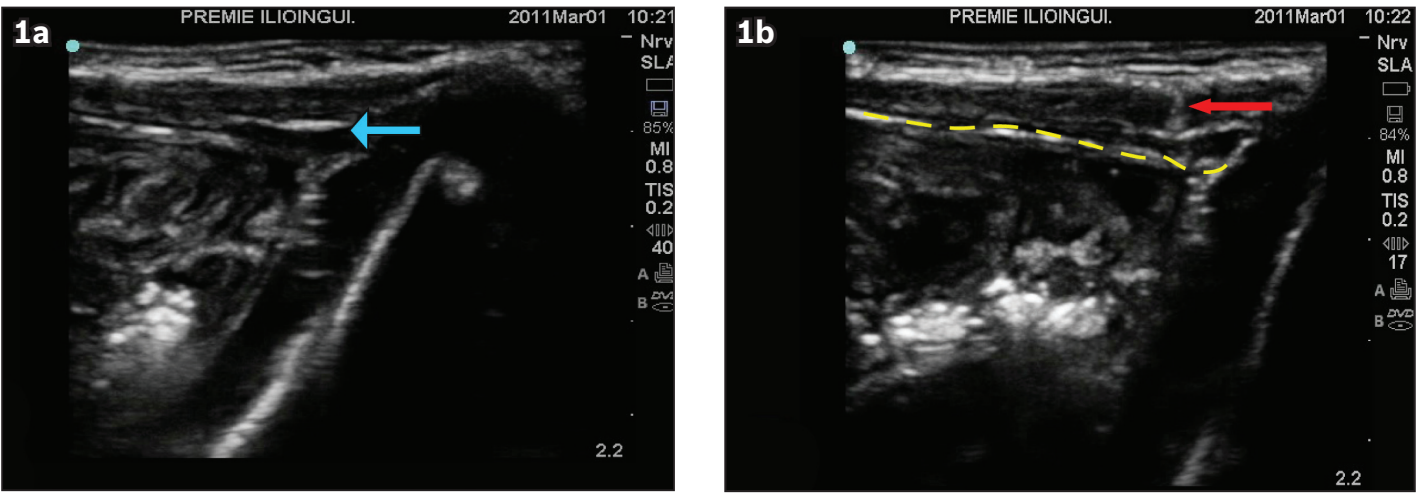

Fig. 1 Ultrasonographic images show (a) the barely seen ilioinguinal-iliohypogastric nerves (blue arrow) between the internal oblique and transversus abdominis muscle; and (b) the needle (red arrow) being inserted 'out-of-plane', with the tip 'tenting' the muscle layers where the ilioinguinal-iliohypogastric nerves lay. The peritoneum (dotted line) lies just beneath the thin muscle layers, over the bowel which moves with respiration.
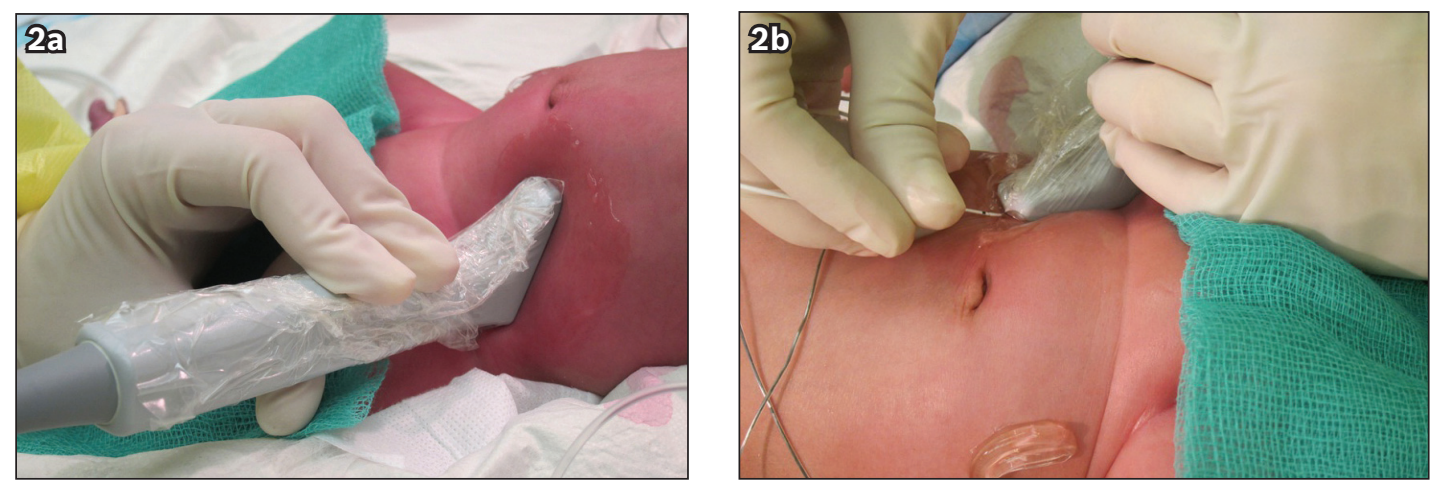

Fig. 2 Photographs show the orientation of the (a) ultrasonography probe; and (b) needle to probe (in-plane approach) during the ultrasonography-guided ilioinguinal-iliohypogastric nerve block.

supplementary oxygen support. Only neonates suitable for general anaesthesia were included. All neonates received general inhalational induction with $6 \%$ sevoflurane in $100 \%$ oxygen. The patients were intubated after paralysis with $0.5 \mathrm{mg} / \mathrm{kg}$ intravenous atracurium, and anaesthesia was maintained using desflurane $6 \%$ in an air/oxygen (50\%/50\%) mixture.

A portable ultrasonography unit (M-turbo; FUJIFILM SonoSite Inc, WA, USA) and a 5-10 MHz linear hockey stick probe was used to identify the transverse view of the IG-IH nerves between the internal oblique and transversus abdominis muscles (Fig. 1a). In most neonates, only the layers were seen, with very subtle or no fascial click even when the layers to be pierced were visualised on ultrasonography. Following aseptic preparation of the puncture site and probe, a short, bevelled, 25G 25-mm needle (BBraun, Melsungen, Germany) was inserted out-of-plane, with its tip relative to the visualised IG-IH nerve/muscle layers (Fig 1b). Fig. 2 shows the orientations of the ultrasonography probe and needle during the procedure.

$0.25 \%$ bupivacaine (up to $0.25 \mathrm{~mL} / \mathrm{kg}$ ) was injected, with an aim to surround both nerves (if visible), and the total amount of LA used was recorded. An average of $0.17 \mathrm{~mL} / \mathrm{kg}$ of $0.25 \%$ bupivacaine was administered on each side, with recorded minimum and maximum doses of $0.10 \mathrm{~mL} / \mathrm{kg}$ and $0.25 \mathrm{~mL} / \mathrm{kg}$, respectively. All blocks were performed by two paediatric anaesthetists who had experience in ultrasonography-guided IG-IH blocks in older children. The anaesthetists found that manipulation of the ultrasonography probe in premature neonates was more difficult technically due to their distended abdomens. The mean duration of surgery was 54.3 mins.

Failure was defined as the need for intraoperative or postoperative intravenous fentanyl supplementation, and its incidence was recorded. Intravenous fentanyl supplementation was administered intraoperatively if the maximum heart rate was $>20 \%$, and it was administered postoperatively if Neonatal Infant Pain Scale (NIPS) score was $>6$. NIPS was scored every 10 mins in the post-anaesthesia care unit for 30 mins. ${ }^{(10)}$ The block was successful in all six patients, with no patient requiring intravenous fentanyl as 'rescue analgesic' intraoperatively or postoperatively. Transient tachycardia during peritoneal traction and manipulation of the spermatic cord was encountered, but this was resolved without additional intravenous opioids. The highest NIPS score recorded was 2; this score decreased to 0 with waddling and holding.

Surgeon satisfaction was high for all six patients, with special mention that the minimal LA volume used ensured that surgical planes were not disrupted. No complications of peritoneal puncture or femoral nerve palsy occurred. All six neonates had uneventful postoperative recovery, with no postoperative apnoea. None of the neonates required postoperative ventilation. 


\section{DISCUSSION}

We found that ultrasonography-guided IG-IH blocks for inguinal herniotomies can be performed safely and efficaciously in premature neonates. This procedure is able to achieve effective opioid-free analgesia using low volumes of LA. The mean LA volume used in our series was $0.17 \mathrm{~mL} / \mathrm{kg}$, while it was $0.25 \mathrm{~mL} / \mathrm{kg}$ for procedures using the conventional landmark-based technique. ${ }^{(4)}$ The use of low volumes of LA help to reduce the potential complications of LA toxicity in neonates, who have lower concentrations of alpha-1 acid glycoprotein. In addition, lower volumes of LA deposited in between the muscle layers lead to less disruption of surgical planes, thereby improving operating conditions.

Herein, we report the case of six ex-premature neonates, for whom ultrasonography-guided IG-IH nerve blocks were successfully performed for inguinal herniotomies, achieving opioid-free analgesia and safe extubation after general anaesthesia. This technique is a feasible and valuable option for anaesthetising apnoea-prone preterm neonates presenting for inguinal herniotomies who may have contraindications for neuraxial blockade.

\section{REFERENCES}

1. Williams JM, Stoddart PA, Williams SA, Wolf AR. Post-operative recovery after inguinal herniotomy in ex-premature infants: comparison between sevoflurane and spinal anaesthesia. Br J Anaesth 2001; 86:366-371.

2. Schwartz D, Al-Najjar H, Connelly NR. Caudal block in a child with a sacral dimple utilizing ultrasonography. Pediatr Anesth 2011; 21:1073-4.

3. Ecoffey C, Lacroix F, Giaufre E, et al. Epidemiology and morbidity of regional anaesthesia in children: a follow-up one-year prospective survey of the French-Language Society of Paediatric Anesthesiologists (ADARPEF). Pediatr Anesth 2010; 20:1061-9.

4. Thong SY, Lim SL, Ng SB. Retrospective review of ilioinguinaliliohypogastric nerve block with general anesthesia for herniotomy in ex-premature neonates. Pediatr Anesth 2011; 21:1109-13.

5. Van Schoor A, Bosman MC, Bosenberg AT. Anatomical considerations of the neonatal ilioinguinal/iliohypogastric nerve block (poster). South Africa: University of Pretoria, 2009.

6. Weintraud M, Marhofer P, Bosenberg A, et al. Ilioinguinal / iliohypogastric blocks in children: where do we administer the local anesthetic without direct visualization? Anesth Analg 2008; 106: 89-93.

7. Lim SL, Ng Sb A, Tan GM. Ilioinguinal and iliohypogastric nerve block revisited: single shot versus double shot technique for hernia repair in children. Paediatr Anaesth. 2002; 12:255-60.

8. Willschke H, Marhofer P, Bosenberg A, et al. Ultrasonography for ilioinguinal/iliohypogastric nerve blocks in children. Br J Anaesth 2005; 95:226-30

9. Willschke $\mathrm{H}$, Bosenberg A, Marhofer P, et al. Ultrasonographic-guided ilioinguinal/iliohypogastric nerve block in pediatric anesthesia: what is the optimal volume? Anesth Analg 2006; 102:1680-4.

10. Suraseranivongse $\mathrm{S}$, Kaosaard R, Intakong $\mathrm{P}$, et al. A comparison of postoperative pain scales in neonates. Br J Anaesth 2006; 97:540. 